\title{
WHICH OPERATORS ARE SIMILAR TO PARTIAL ISOMETRIES?
}

\author{
L. A. FIALKOW
}

\begin{abstract}
Let $\mathcal{X}$ denote a separable, infinite dimensional complex Hilbert space and let $\mathscr{E}(\mathcal{H})$ denote the algebra of all bounded linear operators on $\mathscr{T}$. Let $\mathscr{P}=\{T$ in $\mathcal{E}(\mathcal{H}) \mid r(T)<1$ and $T$ is similar to a partial isometry with infinite rank $\}$; let $\mathcal{S}=\{S$ in $\mathcal{L}(\mathcal{C}) \mid r(S)<1, \operatorname{range}(S)$ is closed, and $\operatorname{rank}(S)$ $\left.=\operatorname{nullity}(S)=\operatorname{corank}(S)=\kappa_{0}\right\}$. It is conjectured that $\mathscr{P}=\mathcal{S}$ and it is proved that $\mathcal{P} \subset \delta \subset \mathcal{P}^{-}$.
\end{abstract}

Introduction. Let $\mathcal{H}$ denote a fixed separable, infinite-dimensional complex Hilbert space, and let $\mathcal{L}(\mathcal{F C})$ denote the algebra of all bounded linear operators on $\mathcal{H}$. In [5], Sz.-Nagy proved that an invertible operator $T$ in $\mathscr{L}(\mathcal{H})$ is similar to a unitary operator if and only if the powers of $T$ and $T^{-1}$ are uniformly bounded; the proof of this result also implies that an operator is similar to an isometry if and only if its powers are uniformly bounded above and below [4]. In this note we state the following conjecture concerning operators similar to partial isometries, and then prove results which partially affirm the conjecture.

ConJecture. If $T$ is an operator on $\mathcal{H}$ with closed range, whose spectral radius is less than one, and such that $\operatorname{rank}(T)=\operatorname{nullity}(T)=\operatorname{nullity}\left(T^{*}\right)$ $=\aleph_{0}$, then $T$ is similar to a partial isometry.

Let $\mathcal{P}=\{T$ in $\mathscr{L}(\mathcal{C}) \mid r(T)<1$ and $T$ is similar to a partial isometry with infinite rank $\}$, where $r(T)$ is the spectral radius of $T$; let $\mathcal{S}=\{S$ in $\mathcal{L}(\mathcal{H}) \mid r(S)$ $<1$, $\operatorname{range}(S)$ is closed, and $\left.\operatorname{rank}(S)=\operatorname{nullity}(S)=\operatorname{corank}(S)=\aleph_{0}\right\}$. It is easy to prove that $\mathscr{P} \subset \delta$ and in this note we prove that $\mathscr{P} \subset \delta \subset \mathscr{P}^{-}$(the norm closure of $\mathcal{P}$ in $\mathcal{L}(\mathcal{H})$ )). To state the results in detail we use the following notation. If $A$ and $B$ are operators on $\mathcal{H}$ such that $A^{*} A+B^{*} B$ is invertible, let $M(A, B)$ denote the operator on $\mathcal{H} \oplus \mathcal{H}$ whose matrix is $\left(\begin{array}{ll}0 & A \\ 0 & B\end{array}\right)$; let $\mathcal{T}$ denote the set of all matrices of this form whose spectral radii are less than one. Each operator in $\mathcal{S}$ is unitarily equivalent to a matrix in $\mathcal{T}$.

THEOREM 1. The operator $M(A, B)$ in $\mathrm{T}$ is similar to a partial isometry if any of the following conditions are satisfied:

(i) 0 is not in the interior of $\sigma(A)$;

(ii) $\operatorname{nullity}(A)=\operatorname{corank}(A)$;

(iii) nullity $(A)<\operatorname{corank}(A)=\aleph_{0}$ and $B$ is not compact;

(iv) $B$ is a semi-Fredholm operator;

(v) $\operatorname{corank}(A)<\operatorname{nullity}(A), A$ has closed range, and $B^{*} \mid E$ is not compact, where

$$
E=\left\{y \in \mathscr{H} \mid \exists x \in \mathcal{H} \ni: A^{*} x+B^{*} y=0\right\} .
$$

Received by the editors October 7, 1974 and, in revised form, January 10, 1975.

AMS (MOS) subject classifications (1970). Primary 47B15.

(C) American Mathematical Society 1976 
Let $(J)$ denote the ideal of all compact operators in $\mathcal{L}(\mathcal{H} \oplus \mathcal{H})$. If $T$ is in $\mathcal{L}(\mathcal{H} \oplus \mathscr{H})$, let $\tilde{T}$ denote the image of $T$ under the canonical homomorphism of $\mathfrak{L}(\mathcal{H} \oplus \mathcal{H})$ on to the Calkin algebra $\mathfrak{L}(\mathcal{H} \oplus \mathcal{H}) /(J)$.

THEOREM 2. If $T=M(A, B)$ is in $\mathcal{T}$ then $\tilde{T}$ is similar to a partial isometry if either of the following conditions is satisfied:

(i) $\operatorname{nullity}(A)$ and corank $(A)$ are finite;

(ii) $B$ is compact.

Because these results do not cover the case corank $(A)<\operatorname{nullity}(A)=\aleph_{0}$, we do not know whether $\mathscr{P}=\delta$. We note also that the proof of Theorem 1-iii was motivated by the proof of a factorization theorem of R. G. Douglas [1, Lemma 2.1]. The author thanks the referee for suggestions that have clarified certain points in the original proofs of our results.

\section{Proof of Theorems 1 and 2.}

Lemma 0 . If $T=M(A, B)$ is in $\mathcal{T}$, then the nonzero elements of $\sigma(T)$ and $\sigma(B)$ are identical.

Proof. If $\lambda \neq 0$ and $B-\lambda$ is invertible, then a calculation shows that $(T-\lambda)^{-1}$ is given by the operator matrix

$$
\left(\begin{array}{cc}
-1 / \lambda & (-1 / \lambda) A(B-\lambda)^{-1} \\
0 & (B-\lambda)^{-1}
\end{array}\right)
$$

If $\lambda \neq 0$ and the inverse of $T-\lambda$ exists, denote this inverse by the operator

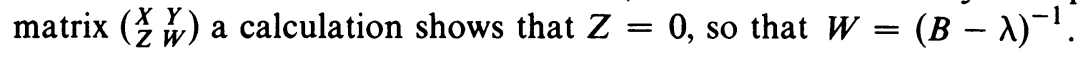

Lemma 1. If $T$ is in $\mathcal{T}$, then $T$ is similar to an operator $M(A, B)$ such that $\|B\|<1$.

Proof. If $T=M(A(T), B(T))$, Lemma 0 implies that $r(B(T))<1$, and Problem 122 of [3] implies that there exists an invertible operator $X$ such that $\left\|X B(T) X^{-1}\right\|<1$. Since $T$ is similar to $M=M\left(A(T) X^{-1}, X B(T) X^{-1}\right)$, the proof is complete.

Lemma 2. If $T$ is in $\mathcal{T}$ and nullity $(A(T))=$ corank $(A(T))$, then $T$ is similar to an operator $M(A, B)$ such that $A \geqq 0$ and $\|B\|<1$.

Proof. Consider the operator $M$ of Lemma 1. We have nullity $\left(A(T) X^{-1}\right)$ $=\operatorname{nullity}(A(T))=\operatorname{corank}(A(T))=\operatorname{corank}\left(A(T) X^{-1}\right)$, and thus $A(T) X^{-1}$ $=U P$, where $U$ is unitary and $P \geqq 0$. Since $M$ is unitarily equivalent to $M\left(P, X B(T) X^{-1}\right)$, the proof is complete.

Lemma 3. Let $T=M(A, B)$ be in $\mathcal{T}$ and suppose $A^{*} A+B^{*} B \geqq \varepsilon^{2}>0$. If $|\lambda|>1$, then $T$ is similar to $M(A-\varepsilon / \lambda, B)$.

Proof. Theorem 1 of [1] implies that there exist operators $X_{1}$ and $X_{2}$ such that $X_{1} A+X_{2} B=\varepsilon$ and $X_{1}^{*} X_{1}+X_{2}^{*} \cdot X_{2} \leqq 1$. Let $|\lambda|>1$ and let $S$ denote the operator on $\mathcal{H} \oplus \mathcal{H}$ whose matrix is 


$$
\left(\begin{array}{cc}
X_{1}-\lambda & X_{2} \\
0 & -\lambda
\end{array}\right) \text {. }
$$

Now $S$ is invertible and a calculation shows that $\operatorname{SM}(A, B) S^{-1}$ is of the desired form.

Proof of Theorem 1-i. The operator $M$ of Lemma 1 is similar to $M\left(X A(T) X^{-1}, X B(T) X^{-1}\right)$, and thus we may assume that $\|B\|<1$ and 0 is not in the interior of $\sigma(A)$. By an application of Lemma 3 with $\lambda$ suitably chosen such that $A-\varepsilon / \lambda$ is invertible and $|\lambda|>1$, we may assume that $A$ is invertible. Since $\|B\|<1$, we may define $R=A\left(1-B^{*} B\right)^{-1 / 2}$ and $S=R$ $\oplus 1_{\mathcal{K}}$; a calculation shows that $S^{-1} T S=M\left(\left(1-B^{*} B\right)^{1 / 2}, B\right)$, which is a partial isometry, and therefore the proof is complete.

Proof of Theorem 1-ii. We may assume from Lemma 2 that $A \geqq 0$; the result now follows from Theorem 1-i.

Proof of Theorem 1-iii. Recall that an operator $B$ in $\mathcal{L}(\mathcal{H})$ is not compact if and only if the range of $B$ contains a closed, infinite-dimensional subspace (see, for example, Theorem 2.5 of [2] and Problem 141 of [3]). It follows from this fact and an application of the open mapping theorem that $B$ is not compact if and only if $B$ is bounded below on some closed, infinitedimensional subspace $M \subset \operatorname{ker}(B)^{\perp}$. Thus there exists $\delta>0$ such that $\|B m\| \geqq \delta\|m\|$ for all $m$ in $M$. For each $m$ in $M$, we set $X_{1}(B m)=A m$. Now

$$
\left\|X_{1}(B m)\right\|=\|A m\| \leqq\|A\|\|m\| \leqq(\|A\| / \delta)\|B m\|,
$$

and it follows that $X_{1}$ is a well-defined bounded linear operator defined on the closed subspace $B(M)$. Let $Q$ denote the projection onto $B(M)$, and let $X=X_{1} Q$ in $\mathcal{L}(\mathcal{H})$. Now $M \subset \operatorname{ker}(A-X B)$ and since $(A-X B) \mathcal{H} \subset A \mathcal{H}$, we have $\operatorname{dim} \operatorname{ker}(A-X B)=\operatorname{dim} \operatorname{ker}\left((A-X B)^{*}\right)=\aleph_{0}$. Since $T$ is similar to $M(A-X B, B)$, the proof may be completed by an application of Theorem 1ii.

Proof OF Theorem 1-iv. From Lemma 1, we may assume $\|B\|<1$. Recall that an operator $B$ in $\mathcal{L}(\mathcal{H})$ is semi-Fredholm if $B$ has closed range and if either nullity $(B)$ or corank $(B)$ is finite. We consider first the case nullity $(B)<\aleph_{0}$; there exists an operator $L$ and a finite rank operator $K$ such that $L B=1+K$. Let $X=\left(\sqrt{1-B^{*} B}-A\right) L$ and let $S$ denote the operator on $\mathcal{H} \oplus \mathcal{H}$ whose matrix is $\left(\begin{array}{ll}1 & X \\ 0 & 1\end{array}\right)$. A calculation shows that $S T S^{-1}=M\left(\sqrt{1-B^{*} B}+J, B\right)$, where $J$ is a finite rank operator. Since $\|B\|<1, \sqrt{1-B^{*} B}+J$ is Fredholm with index equal to zero, and the proof may be completed by an application of Theorem 1-ii.

We now consider the case corank $(B)<\boldsymbol{\aleph}_{0}$. In this case $B^{*}$ has finite nullity and closed range. Let $P$ denote the projection onto the initial space of $B^{*}$ and let $\mathcal{E}=\left\{x \in \mathcal{K} \mid \exists y \in P \mathcal{H}\right.$ such that $\left.A^{*} x+B^{*} y=0\right\}$. Since $B^{*}$ has closed range, $\mathscr{E}$ is closed; since nullity $\left(T^{*}\right)=\boldsymbol{\aleph}_{0}$ and nullity $\left(B^{*}\right)<\boldsymbol{N}_{0}$. It follows readily that $\mathscr{E}$ is infinite dimensional. For each $x$ in $\mathscr{E}$ there is a unique vector $X_{1}(x)$ in $P \mathcal{H}$ such that $A^{*} x+B^{*} X_{1}(x)=0$. Since $B^{*}$ is bounded below on $P^{\mathcal{H}}$, the assignment $x \rightarrow X_{1}(x)$ is bounded and linear on the closed subspace $\mathcal{E}$. Let $Q$ denote the projection onto $\mathcal{E}_{1}$ and let $X=X_{1} Q$ in $\mathcal{E}(\mathcal{H})$; thus $\mathcal{E} \subset$ $\operatorname{ker}\left(A+X^{*} B^{*}\right)^{*}$. Since $\mathcal{E}$ is infinite dimensional and $B$ is not compact, the proof may be completed by an application of Theorem 1-ii-iii. 


\section{Corollary. $\mathcal{T} \subset \mathcal{P}^{-}$.}

Proof. The preceding result implies that if $T$ is in $\mathscr{T}$ and $B(T)$ is either left or right invertible, then $T$ is in $\mathscr{P}$. Now there exists a sequence $\left\{B_{k}\right\} \subset \mathfrak{L}(\mathcal{F})$ such that $\lim \left\|B_{k}-B(T)\right\|=0$ and such that the sequence elements are either all left invertible or all right invertible [3, Problem 109]. Since $B_{k}^{*} B_{k}+A^{*} A$ $\rightarrow B^{*} B+A^{*} A$, we may assume that each $B_{k}^{*} B_{k}+A^{*} A$ is invertible; from the upper semicontinuity of the spectrum we may assume each $r\left(B_{k}\right)<1$. Therefore, Theorem 1-iv implies that each $M\left(A, B_{k}\right)$ is in $\mathcal{P}$, and the proof is complete.

We now assume that $T$ is in $\mathcal{T}$ and that $A^{*}$ has closed range and finite nullity. Let $E$ be as in Theorem 1-v; the hypotheses imply that $E$ is a closed, infinite-dimensional subspace. In view of the previous results it is natural to attempt to find an operator $X$ such that corank $(A+X B)=\aleph_{0}$; the following result proves Theorem 1-v.

Proposition. There exists an operator $X$ such that $\operatorname{corank}(A+X B)=\aleph_{0}$ if and only if $B^{*} \mid E$ is not compact.

Proof. If $B^{*} \mid E$ is not compact, the operator $X$ may be constructed by a straightforward modification of the proof of Theorem 1-iii; details are omitted.

For the converse, we assume that $B^{*} \mid E$ is compact. Suppose that there is an operator $X$ on $\mathcal{H}$ and a closed, infinite-dimensional subspace $K \subset \mathcal{H}$ such that $A^{*} t=B^{*} X^{*} t$ for each $t$ in $K$. Since $\operatorname{dim} \operatorname{ker}\left(A^{*}\right)<\aleph_{0}$, it follows that $L=K \cap \operatorname{range}(A)$ is infinite dimensional. Since $A^{*}$ has closed range, $A^{*}$ is bounded below on $L$. Let $\left\{t_{n}\right\}$ denote an orthonormal basis for $L$. Now $t_{n} \stackrel{w}{\longrightarrow} 0,\left\{X^{*}\left(t_{n}\right)\right\} \subset E$, and thus $B^{*} X^{*} t_{n} \rightarrow 0$. Therefore $A^{*} t_{n} \rightarrow 0$, which is a contradiction.

Proof of Theorem 2-i. Let $A=U P$ denote the polar decomposition of $A$. Since $P^{2}+B^{*} B$ is invertible, we may define $T_{1}=M(P, B)$, and Lemma 0 implies that $r\left(T_{1}\right)=r(B)=r(M(A, B))<1$. Theorem 1-ii now implies that $T_{1}$ is similar to a partial isometry. Since the nullity and corank of $U$ are finite, $\tilde{U}$ is unitary, and the proof is completed by noting that

$$
T_{1}-\left(U^{*} \oplus 1\right) T(U \oplus 1)
$$

is of finite rank.

Proof of Theorem 2-ii. Theorem 1 of [1] implies that there exist operators $X_{1}$ and $X_{2}$ such that $X_{1} A+X_{2} B=1$. Since $B$ is compact, we have $\tilde{X}_{1} \tilde{A}=1$, and thus $A$ has closed range and finite nullity. If $A=U P$ denotes the polar decomposition of $A$, then $P=Q \oplus 0$, where $Q$ is invertible. Set $R=Q^{-1}$ $\oplus 1_{\operatorname{ker}(P)}$ and $S=1_{\mathcal{C}} \oplus R$. Now $S^{-1} T S$ has the operator matrix

$$
\left(\begin{array}{cc}
0 & U \\
0 & R^{-1} B R
\end{array}\right)
$$

which is the sum of a partial isometry and a compact operator. 


\section{REFERENCES}

1. R. G. Douglas, P. S. Muhly and Carl Pearcy, Lifting commuting operators, Michigan Math. J. 15 (1968), 385-395. MR 38 \#5046.

2. P. A. Fillmore and J. P. Williams, On operator ranges, Advances in Math. 7 (1971), 254-281. MR 45 \# 2518.

3. P. R. Halmos, A Hilbert space problem book, Van Nostrand, Princeton, N. J., 1967. MR 34 \#8178.

4. - Ten problems in Hilbert space, Bull. Amer. Math. Soc. 76 (1970), 887-933. MR 42 \#5066.

5. B. de Sz.-Nagy, On uniformly bounded linear transformations in Hilbert space, Acta Univ. Szeged. Sect. Sci. Math. 11 (1947), 152-157. MR 9, 191.

Department of Mathematics, Western Michigan University, Kalamazoo, Michigan 49001 